\title{
Analysis of Steady-State Transients in Distributed Low- Voltage Power Systems with Rectified Loads
}

\author{
WILLIAM P. GIBBONS, MEMBER. IEEE
}

\begin{abstract}
An analysis of a large number of randomly connected small low-voltage rectifiers to general purpose distribution systems is presented. The primary interest is on single-phase rectifiers connected to $120 / 208 \mathrm{~V}$ three-phase four wire "wye" systems, and shows that the cumulative effect of several different types of rectifiers can produce results which are generally not anticipated. Some of the unanticipated results are overcurrent conditions on the neutral conductor and line conditioning devices which produce effects quite different from those which they are intended to prevent.
\end{abstract}

\section{INTRODUCTION}

$\mathbf{T}$ IHE INSTALLATION of equipment which contains small rectifier power supplies has evidently never presented much of a problem when added to general office, commerical, or industrial low voltage ( $120 \mathrm{~V}$ single phase) distribution systems. If anything, the other equipment on the distribution system is responsible for generating and transmitting disturbances that might interfere with the operation of the equipment that was associated with the rectified power. Without question there is continued growth and expanded use of minicomputers and microprocessors, distributed miniprocessor and microprocessor systems, and other solid-state devices deriving power from rectified power supplies. Hence, the possibility of low-voltage distribution systems having a significant percentage of or being entirely dedicated to rectified supplies is growing and can lead to some unusual results.

This article is the result of a study of unusual conditions existing in the power systems of three facilities, each of which contain a large number of small rectified power supplies. The facilities are in three separate locations and are electrically very far apart. The reason for each system study was independently requested for voltage spikes and power line fluctuations.

The literature on rectification is not generally concerned with the conditions in low-voltage distribution systems. In particular, in reference [1] (only one of several good texts), the usual practice is to use an idealized electromotive force (EMF) as the voltage source whether this be a generator, transformer, or bus anyplace in a distribution system (see Fig. 1). The other type of analysis is generally concerned with the operation of a large rectifier application [2]. In between there are many informative and theoretical articles dealing with

Paper IPSD 79-10, approved by the Power Systems Support Committee of the IEEE Industry Applications Society for presentation at the 1979 Industrial and Commercial Power Systems Technical Conference, Seattle, WA, May 14-17. Manuscript released for publication August 17, 1979.

The author is with the United States Air Force, Directorate of Civil Engineering, Aeronautical Systems Division, Wright-Patterson Air Force Base, $\mathrm{OH} 45433$.

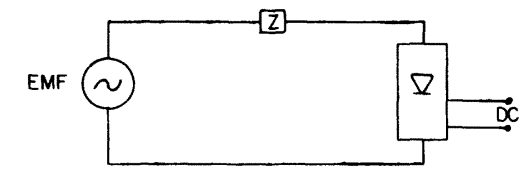

Fig. 1. Typical voltage source for rectifier analysis.

idealized source and single rectifier loads with various complex impedances, e.g., $z$ of Fig. 1, between source and load [3], [4].

In most of the sources mentioned and in many others the presence of internally generated transients in electrical systems with rectifiers is either ignored or indirectly dealt with. What is presented now are some appraisals of general guidelines that have been presented on transient generation and corrective or preventative measures such as

1) analysis of transient generation;

2) the third harmonic of a $60-\mathrm{Hz}$ system becoming the fundamental on the neutral conductor and the resulting neutral current becoming higher than any line current;

3 ) the relation of power system stiffness and the occurrence of disturbances;

4) the effects of isolation transformers with electrostatic shielding.

\section{TRANSIENT GENERATION [5]}

The analysis of the waveforms observed on the power distribution system can begin with a discontinuity or impulse function describing a sudden change $\mu$ independently of the system frequency. In this article, two current-impulse functions are of interest. One is the positive current impulse, defined here as $\mu_{+i}\left(t-T_{1}\right)$, which occurs in the same electrical time $0-\pi$ (see Fig. 2). If the current pulse is negative during the time that the voltage is between $\pi$ and $2 \pi$, then the current is still taken as positive, $\mu_{+i}$ in the sense of going in the same direction as the voltage. The other impulse is the sudden negative current impulse $\mu_{-i}\left(t-T_{2}\right)$ during the positive $(0-\pi)$ voltage excursion (see Fig. 2).

It will be noted that only a portion of the current waveshapes are being shown in Fig. 2. This is due to the fact that only a portion of the total current waveshape undergoes a sudden change, and only in that region $T$ of the sudden change is the present interest involved. The shape before and after the occurrence is governed by steady-state relations and is not involved in the transient generation.

An equivalent circuit for the analysis would be quite complex if all the branch circuits and all the loads were included. 


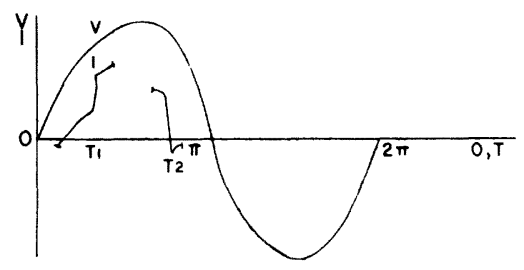

Fig. 2. Definition of positive impulse, $\mu_{+i}\left(t-T_{1}\right)$, and negative impulse, $\mu_{i}\left(t-T_{2}\right)$.

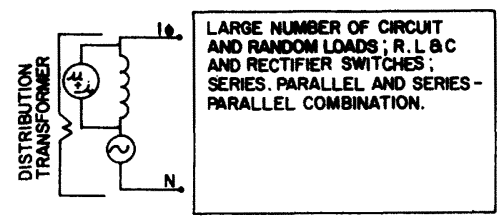

Fig. 3. Components of rectifier loaded distribution system.

What is important is the most significant lumped component in the power circuit which is the inductance of the transformer. In this respect the circuit of Fig. 3 will be used to represent the components and the area of interest which is the circuit from the distribution transformer to the circuits and random loads. An equivalent current source $\mu_{+i}$ is introduced in order to account for both types of impulse. This approach is taken since the rectifier loads can account for the positive impulse through a change of impedance at conduction time. But there must still be a current source to account for the reverse current $\mu_{-i}$ flowing in opposition to the single-phase voltage polarity.

The direction of the current impulses and their effect is shown in Fig. 4 in relation to polarity convention and the actual mode of the voltage spike generation. Fig. 4(a) shows the effect of a positive current impulse $\mu_{+i}$ during the positive voltage interval $(0-\pi)$ and the resultant voltage pulse generation due to the $L(d i / d t)$. Fig. 4(b) shows graphically the current impulse and the resultant voltage waveform at time $T$ which is $L(d i / d t)$ at points $A-B$ in the distribution system. Fig. 4(c) and (d) shows the negative current impulse convention and the resultant voltage waveform $L(d i / d t)$ a short distance from the distribution transformer.

The predominant type of voltage response by the distribution system to the current impulse will generally be the single storage element $L(d i / d t)$. This is due to the inductance $L$ of the transformer which will be much larger than any single device on a branch circuit. The single storage element waveform is the one depicted in Fig. 4(b) and (d). The presence of capacitance, either distributed or lumped, does give rise to the oscillatory condition with large positive and negative excursions superimposed on the sinusoidal voltage waveform. The source of oscillations or ringing is due either to the under-damped series $L R C$ circuit or the over-damped parallel $L R C$ circuit $(83: 5)$. The oscillatory condition was not observed on the fundamental $60-\mathrm{Hz}$ voltage waveform as shown in reference [6]. What was observed, although not expanded on the cathode ray tube (CRT), was an oscillatory wave superimposed on the voltage notch. This unusual condition was due to one or more of the large number of series

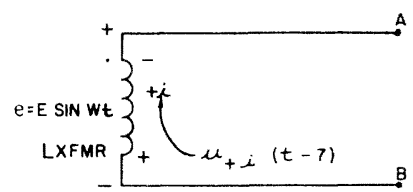

(a)

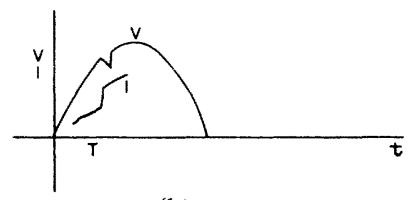

(b)

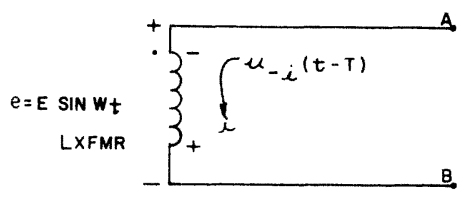

(c)

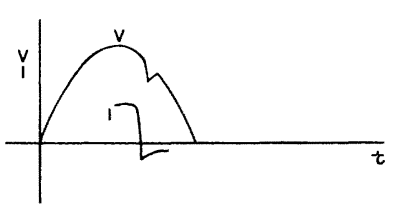

(d)

Fig. 4. Direction of impulse current flow. (a) Positive impulse $\mu_{+i}$. (b) Graphical effect of $\mu_{+i}$. (c) Negative impulse $\mu_{i}$. (d) Graphical effect of $\mu_{-i}$.

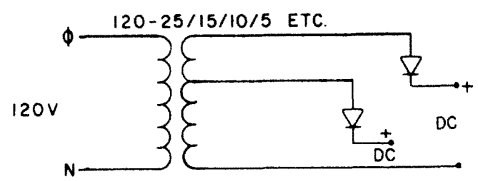

Fig. 5. Typical single-phase rectifier circuits with step down transformers used in all equipment of this study.

impedances and parallel branch circuits being excited during or after the impulse generated voltage across the transformer.

\section{POWER SYSTEM ANALYSIS}

The principal types of rectifiers encountered were the usual variety such as the silicon-controlled rectifier (SCR) and the half-wave and full-wave diode bridge rectifier. The distinguishing feature of the small rectified power supplies of the facilities involved is that they are single phase and derived through stepdown transformers (see Fig. 5). Some equipment had three-phase four-wire connections to distribution panels, but the power supplies were still derived by single phase to neutral connection.

The waveform analysis was done with the Taktronix 7000 series oscilloscopes. This equipment was utilized due to its availability, the low-frequency capability, calibration of the plug-in current amplifier to dc, and the spectrum analyzer (to $10 \mathrm{~Hz}$ ). Any question as to the information displayed, especially on the spectrum display, can be found in the Tektronix literature. 


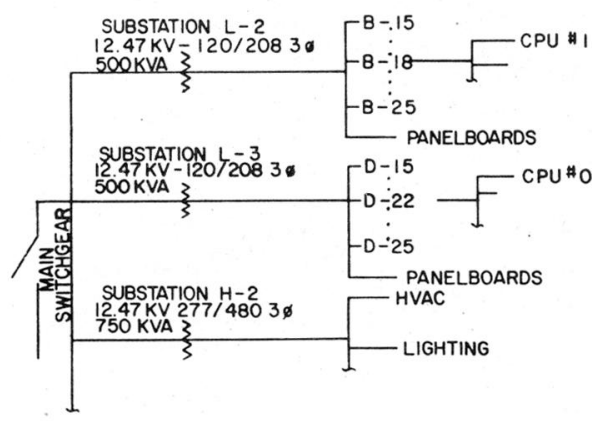

Fig. 6. Single-line diagram of first power distribution system.

\section{A. Distribution System of the First Facility}

The first distribution system under study is shown in Fig. 6 . The facility is supplied by a set of separately derived laboratory bus distribution systems. One half of the building is supplied through the $D$ series risers, and the $B$ series supplies the other half. The laboratory bus system represents practically the entire load on each substation, $L 2$ and $L 3$, with a few panel boards on each having negligible loads.

The observations of the power system consisted of recording the voltage and current waveforms of selected loads and progressing from the loads back through the system to the substations and main switch gear. Along with the analog waveforms, the spectrum of some of the waveforms was recorded in order to determine the magnitude and component harmonic frequencies of any distortions. In the following waveforms the distinctive sine-wave trace is the supply voltage of the particular phase $(A, B$, or $C)$ being observed.

Fig. 7 is the typical voltage and current waveforms of central processing units (CPU) 0 and 1 . The voltage spectra of Fig. 7(b) show the presence of extremely small $(-40 \mathrm{dBV}$ reference) voltage signals in the distortion of the voltage trace of Fig. 7. It is apparent that the voltage distortion is not associated with the inflection points in the current waveforms at this location. Also, the switching phenomena associated with rectification can be seen in the nonsinusoidal current waveform.

Fig. 8 is the sequence of waveforms at riser $B-19$ which includes the load of CPU number 1 (see Fig. 6). Clearly evident at this point is the correspondence between the voltage waveform distortion and the abrupt changes in the current waveform $\mu_{+i}$ (see Fig. 8(a)). The current waveshape is due to the algebraic sum, at that point, of several loads besides CPU number 1 being supplied by riser $B-19$.

The spectrum of the current waveshape, Fig. 8(b), shows the presence of many significant harmonics, through the twenty-fifth at least, on the $+15 \mathrm{dBm}$ reference. Of equal importance is the lack of a corresponding increase in the harmonic content of the voltage waveform (see Fig. 8(c)). The relative amplitudes of the third and fifth harmonics are the same as in Fig. 7(b), but the seventh and fifteenth harmonics have been attenuated between riser $B-18$ and CPU number 1 .

Continuing back through the power system, the next series of waveforms were recorded at the substations and the main switch gear. Fig. 9 is the waveform of the total unibus loads

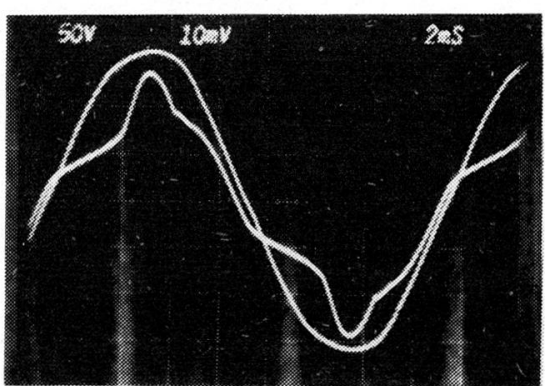

(a)

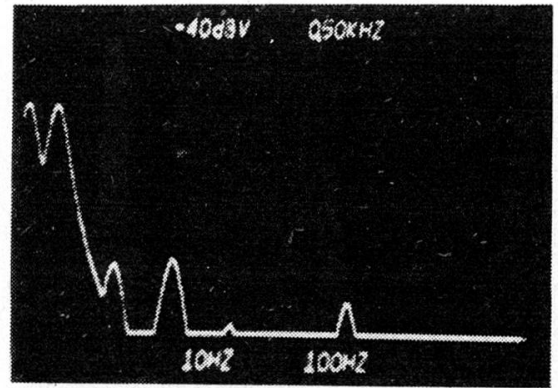

(b)

Fig. 7. Typical waveforms for CPU 0 and 1. (a) Voltage and current. (b) Voltage spectrum with fundamental, third, fifth, seventh, and eleventh harmonic.

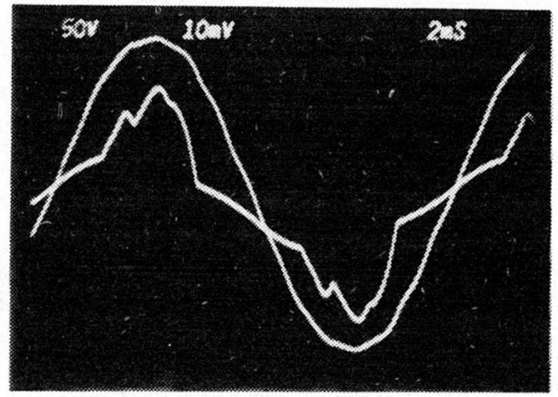

(a)

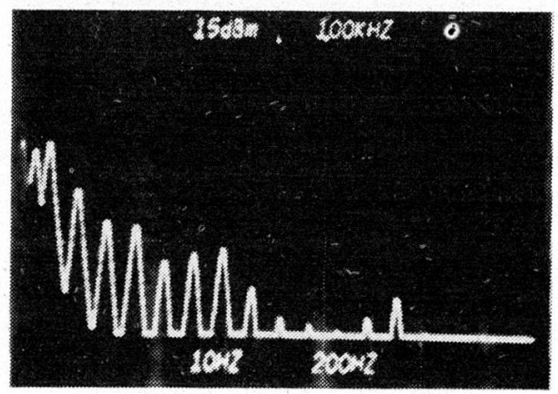

(b)

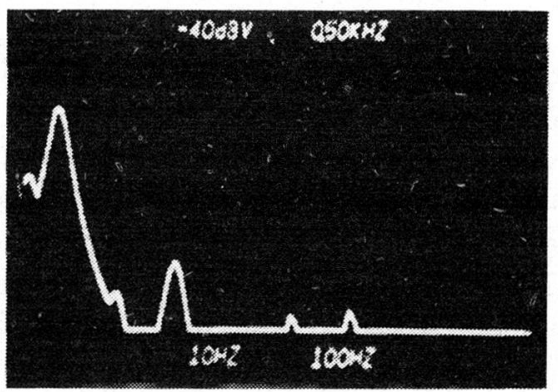

(c)

Fig. 8. Waveforms at riser $B-18$. (a) Voltage and current. (b) Spectrum of current waveform. (c) Sepctrum of voltage. 


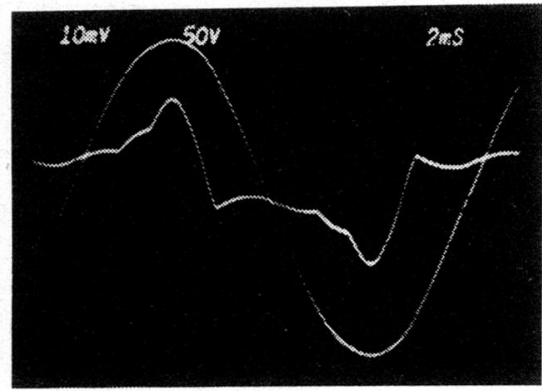

(a)

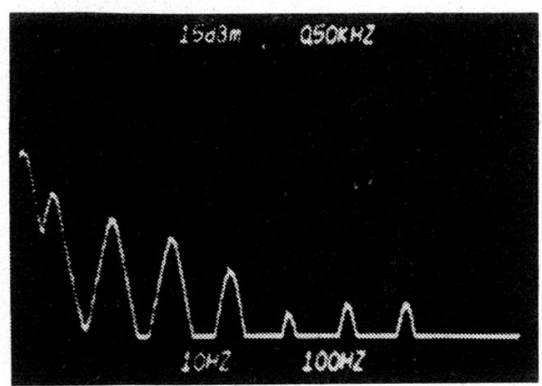

(b)

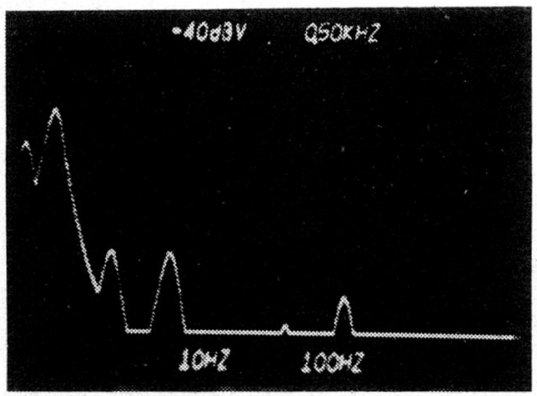

(c)

Fig. 9. Substation $L-2$ waveforms. (a) Voltage and current. (b) Spectrum of current waveform. (c) Spectrum of voltage.

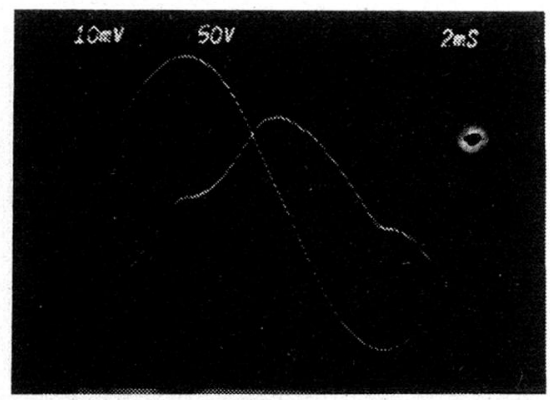

Fig. 10. Substation $H-2$.

on the risers 15-25. Similar waveforms were found on the risers $D$ 15-25.

A further check on the origin of the disturbances was made on substation $H-2$ in Fig. 10 . The load on sub $H-2$ is almost entirely inducive, since it supplies the high-voltage (HV) ac series risers. The voltage waveshape is totally undistorted indicating that the spike is not being propagated into the system on the $12.47 \mathrm{kV}$ system from outside the building nor is the spike being reflected from the low-voltage (LV) (sub $L-2$ and -3) side of the transformer into the $12.47 \mathrm{kV}$ system. The same undistorted waveshape appeared at the main switch gear.
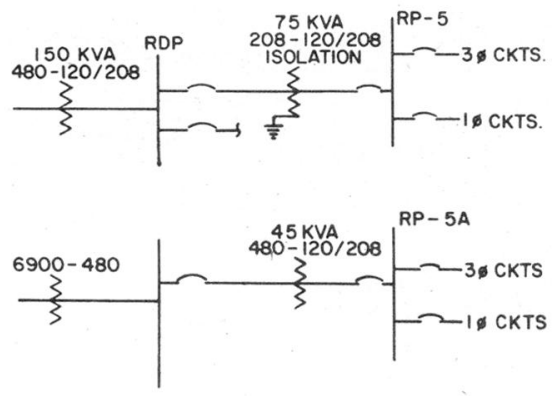

Fig. 11. Single-line diagram of the second facility.

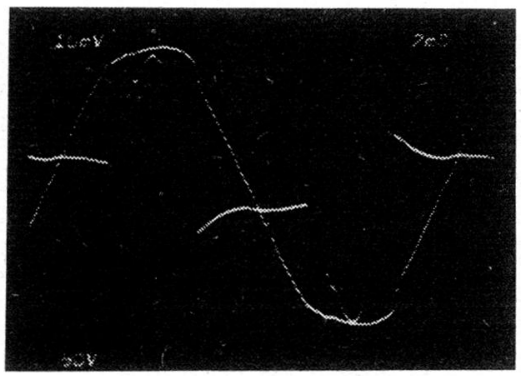

(a)

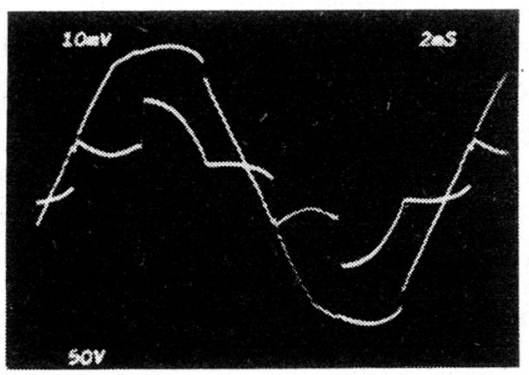

(b)

Fig. 12. Panel RP-5 branch circuit waveforms. (a) DC-DC converter load. (b) SCR load.

\section{B. Distribution System of the Second Facility}

The power system of the second facility is shown in Fig. 11. The procedure for analyzing this power system is similar to that used in the first facility. Sample branch circuit waveforms were recorded. This system had additional intermediate transformers, one isolation and one separately derived, for the distribution system of the respective circuits. These provided a source of additional waveform analysis.

Fig. 12 shows the waveform for two typical branch circuits of panel RP-5 shown in Fig. 11. Fig. 12(a) is the single-phase waveform of a dc-dc converter-type of rectifier, and Fig. 12(b) is the typical SCR current waveform. Fig. 13 is the neutral current of the three-phase branch circuit of which Fig. 12(a) is typical of each independent single-phase load. Using the time base of the voltage signal it is evident that the sum of the narrow single-phase current waveform of each phase is producing a dominant third-harmonic current as the fundamental frequency. The frequency base waveshape of Fig. 13(b) shows the first significant component at $180 \mathrm{~Hz}$ with a slight $60-\mathrm{Hz}$ fundamental due to a small load imbalance.

Fig. 14 shows the feeder conductor currents between panel $R P-5$ and the $75 \mathrm{kVA}$ isolation transformer shown in Fig. 11. 


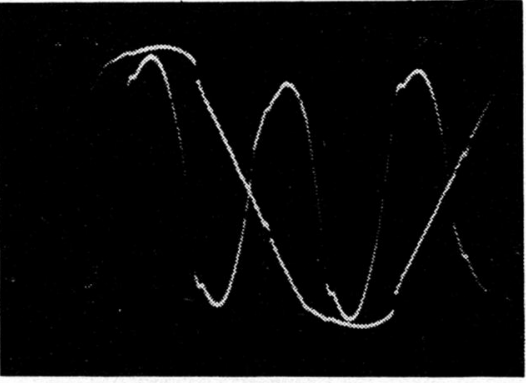

(a)

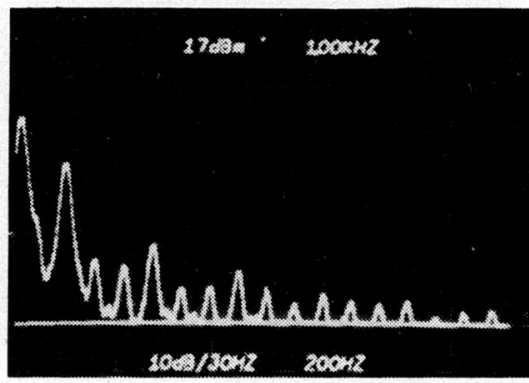

(b)

Fig. 13. DC-DC converter neutral conductor waveform. (a) Phase $A$ voltage and neutral current. (b) Spectrum of current waveform.

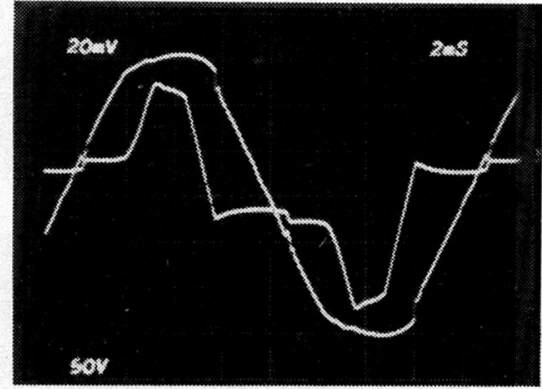

(a)

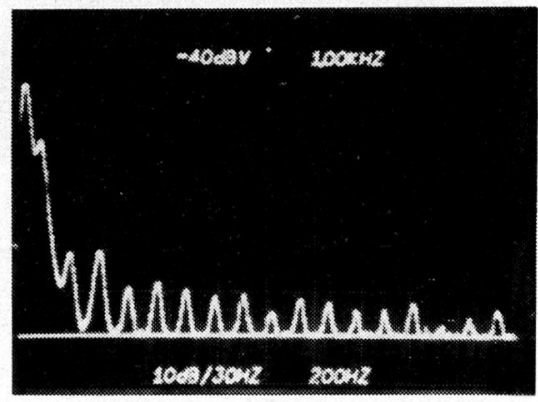

(b)

Fig. 14. Panel RP-5 incoming line waveform. (a) Voltage and current. (b) Spectrum of voltage waveform.

Fig. 14(a) is the phase- $A$ current and is typical for the other two phases. It is clear that the predominant waveshape is composed of devices with Fig. 12(a) waveshapes (approximately 17-500 watt rectifiers per phase) with a small contribution from the SCR. The current sum produces three current step functions; $\mu$ per half cycle, two $\mu_{+i}$, and one $\mu_{-i}$. The step functions at this location are directly related to the voltage distortion points contributed by each load function. Fig. $14(\mathrm{~b})$ is the voltage spectrum indicating the presence of many harmonics which is to be expected from the extremely distorted waveshape.

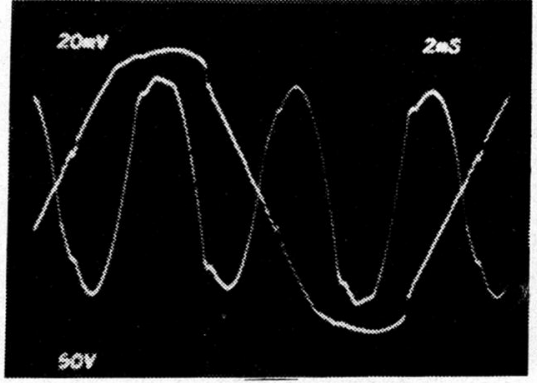

(a)

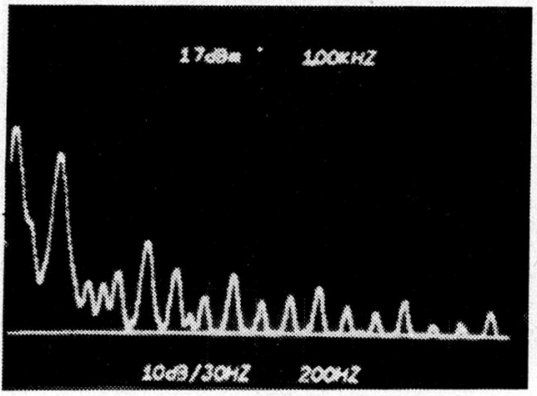

(b)

Fig. 15. Panel RP-5 incoming neutral conductor waveform. (a) Phase $A$ voltage and neutral current. (b) Spectrum of current waveform.

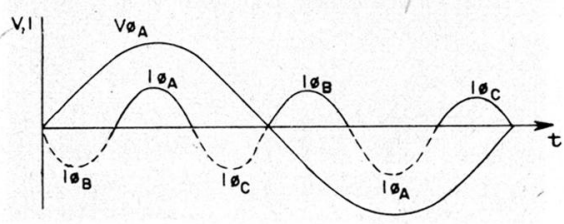

Fig. 16. Graphical development of $180-\mathrm{Hz}$ neutral current generation.

The neutral conductor current for panel $R P-5$ is shown in Fig. 15. It is similar to Fig. 12(a) but with a larger current per division (50 A). The current spectrum, Fig. 15(b), is also similar to Fig. 13(b) with several of the higher harmonics containing more amplitude. This neutral current condition presents a problem in that the effective ( $\mathrm{rms}$ ) value becomes higher than any single-phase current. The single-phase conduction time for the devices occurs between 60 and 120 electrical degrees on the neutral to give the apparent $180 \mathrm{~Hz}$, Fig. 16, instead of cancelling as would happen for three currents of equal magnitude which conduct for a full $360^{\circ}$ cycle. The mechanism of the $180-\mathrm{Hz}$ generation is unusual in this case, although the principle of the cycloconverter $(469: 1)$ shows that it should be expected.

The $180-\mathrm{Hz}$ derived current waveform from a design and safety point of view could be significant in relation to the minimum requirements in neutral wire sizing per National Electrical Code, Articles 215-4 and 220-22. The code specifies neutral sizing for the maximum connected load between any one phase and neutral. The measured value per each phase feeder was $100 \mathrm{~A}$, while the neutral was $140 \mathrm{~A}$ (amprobe and oscilloscope). In this case the three-phase four-wire neutral is 1.4 times the line current which is derived by (7)

$$
\begin{aligned}
I_{\text {eff }} & =\sqrt{I_{\phi A}^{2}+I_{\phi B}^{2}+I_{\phi C}^{2}} \\
& =I \phi \sqrt{3} \quad \text { (maximum possible) } .
\end{aligned}
$$




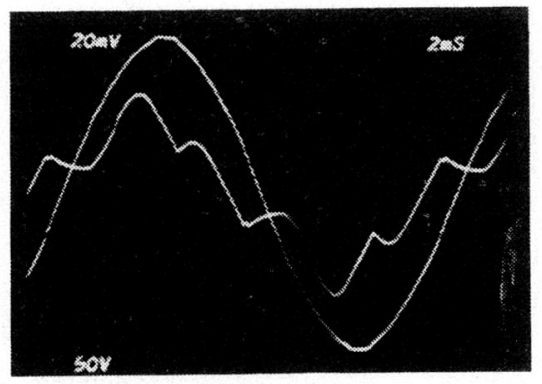

(a)

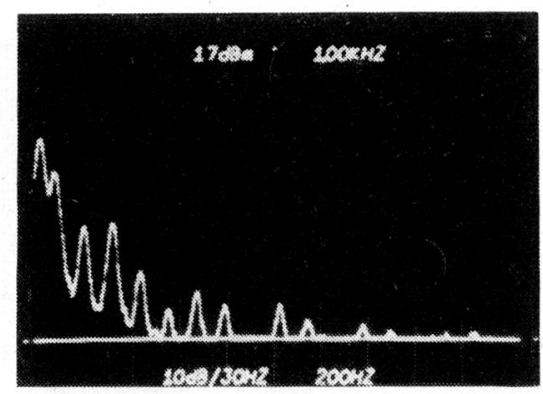

(b)

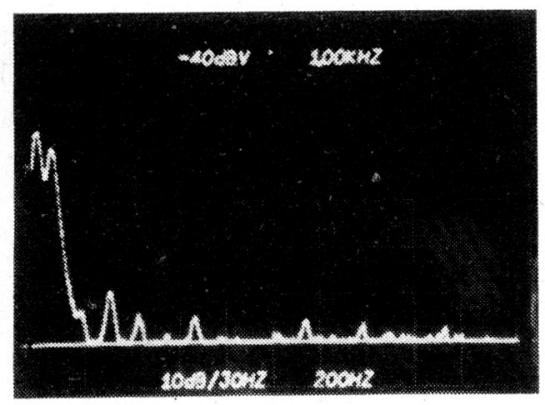

(c)

Fig. 17. Power panel $R D P-2$ waveforms. (a) Voltage and current. (b) Spectrum of current waveform. (c) Spectrum of voltage.

(The code recognizes 140 percent for five-wire two-phase systems but that is not the case here.) Similar values were found on the three-phase branch circuits phase and neutral conductors, Figs. 12(a) and 13(a).

That the current on the neutral conductor was not $171 \mathrm{~A}$ (at $100 \mathrm{~A}$ per phase) was due to the conduction time which was greater than optimum for the $180 \mathrm{-Hz}$ waveform generation. In order for the full three factor to become dominant the conduction time must be $60^{\circ}$ or less in the center of the half cycle on each phase. This will happen when there is no current flow from $0-60^{\circ}$; then maximum conduction from $60^{\circ}-120^{\circ}$; then no current flow from $120^{\circ}$ through $180^{\circ}$. Any current flow greater than $60^{\circ}$ in the center of the half cycle will produce some cancellation, as in this case, where the total neutral current was $140 \mathrm{~A}$.

Continuing on back through the system, Fig. 17 shows the values of the line current and voltage at the power distribution $R D P$-2 which is approximately $150 \mathrm{ft}$ from the isolation transformer. The voltage waveshape is more nearly sinusoidal with many harmonics either eliminated or significantly reduced, as seen in Fig. 17(c) versus Fig. 14(b). The current waveshape is also greatly modified but still contains two impulse functions $\mu_{-i}$ which are responsible for two discernable voltage notches.

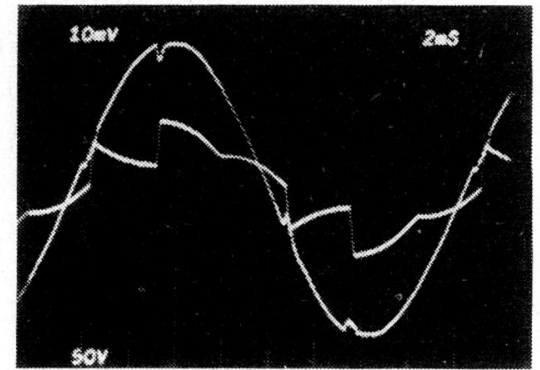

(a)

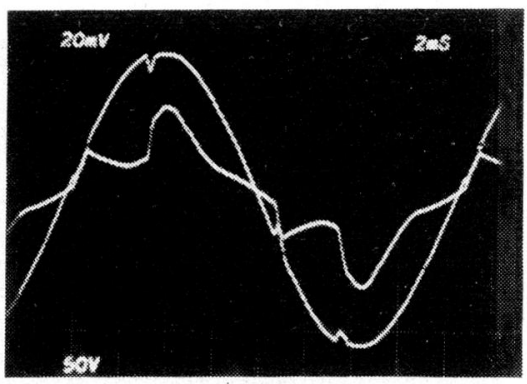

(b)

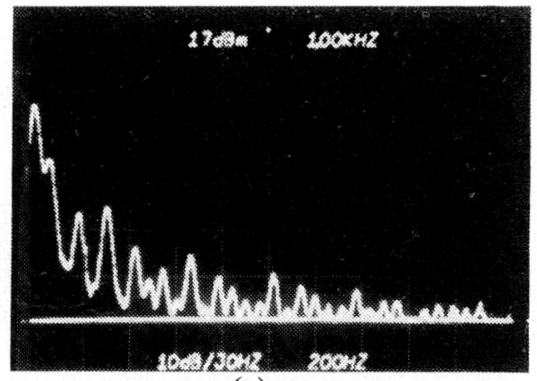

(c)

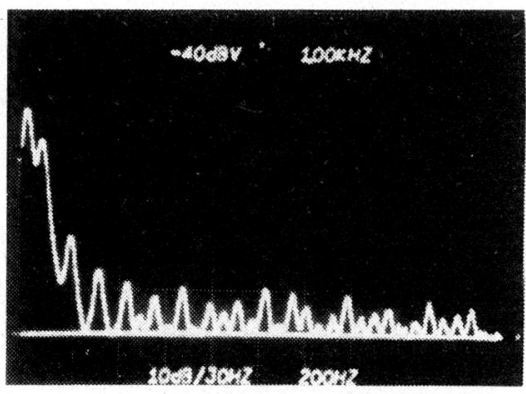

(d)

Fig. 18. Panel $R P-5$ a waveforms. (a) One branch circuit voltage and current. (b) One phase incoming line voltage and current. (c) Incoming line current spectra. (d) Phase voltage spectra.

The second power system (Fig. 11(b)) analysis is shown in Fig. 18. The majority of the loads are SCR with Fig. 18(a) typical of one of the branch loads. The feeder conductors for $R P$-5a, Fig. 18(b), shows the total current waveshape of the SCR circuits with other minor loads modifying the typical SCR waveshape. Fig. 18(c) and (d) are the spectra of the current and voltage signals.

\section{Distribution System of the Third Facility}

The third power system supplied a series of large-scale computers, their peripheral equipment, and other rectifiersupplied devices. Not only was the power system separately derived from the rest of the facility, but several of the distribution systems were provided with isolation transformers and electrostatic shielding, as in Fig. 19. In spite of these provi- 


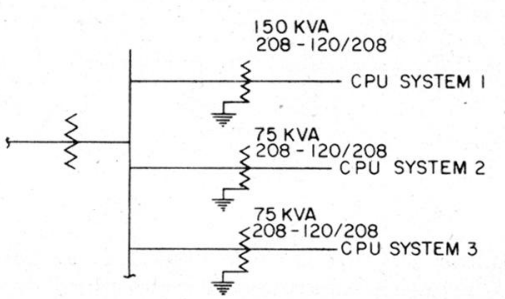

Fig. 19. One-line diagram of the third facility power system.

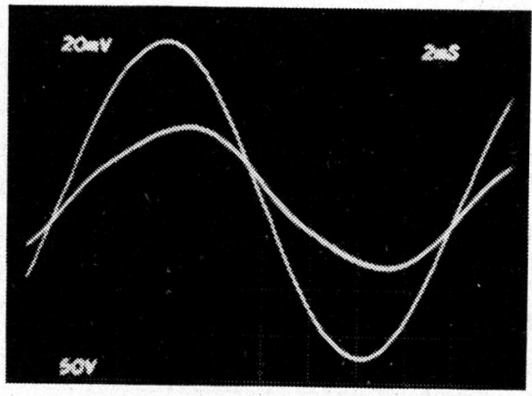

(a)

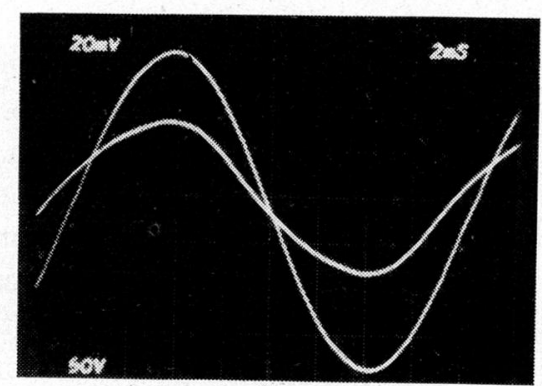

(b)

Fig. 20. Voltage and current waveshapes of CPU system 1. (a) Load side. (b) Line side.

sions the complaints were continuous "spikes" and voltage "fluctuations."

Fig. 20(a) and (b) are the load side and line side waveshapes of the isolation transformer feeding CPU system 1. Both are undistorted, which indicates that the system is neither generating current impulses nor receiving any voltage disturbances. Fig. 21(a) and (b) and Fig. 22(a) and (b) are the typical load side and line side waveshapes of the isolation transformers of CPU systems 2 and 3, respectively. The load side of systems 2 and 3 show significant distortion in the current waveshapes, which in turn cause a corresponding voltage distortion.

However, on the line side of the isolation transformers of systems 2 and 3 much of the voltage distortion has been reduced, although not eliminated. The voltage distortion, which was recorded near the isolation transformer, is localized and is due to impulse currents $\mu$ which are relatively unaffected by the isolation transformer, generating the $L(d i / d t)$ on the line side of the transformers. That this voltage generation is localized can be seen by comparing the voltage waveshape of Figs. 21(b) and 22(b) with the undistorted voltage in Fig. 20(b) and noting that they are all supplied from the same bus in Fig. 19.

\section{DISCUSSION}

The problem with a large number of randomly connected rectifier loads on a low-voltage distribution system is obvious;

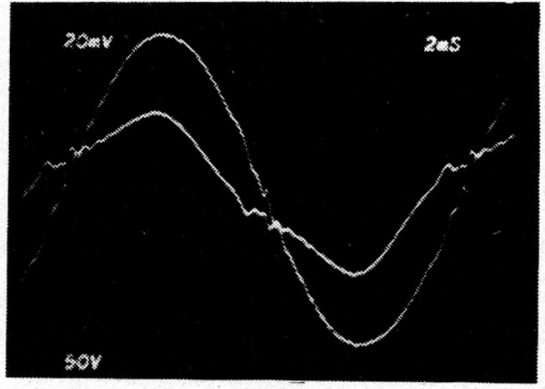

(a)

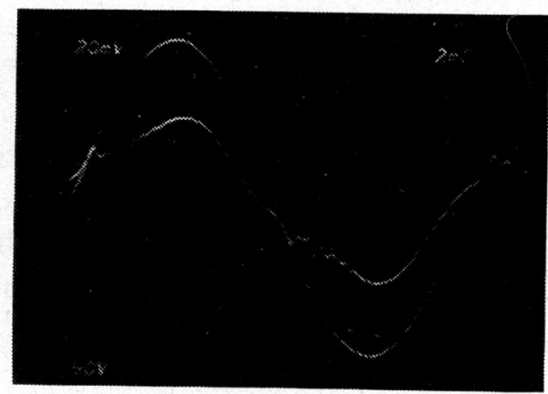

(b)

Fig. 21. Voltage and current waveshapes of CPU system 2. (a) Load side. (b) Line side.

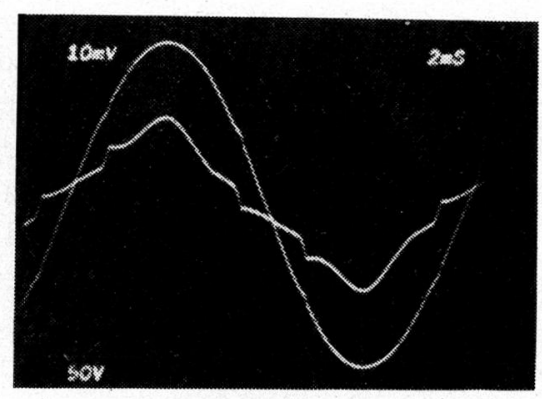

(a)

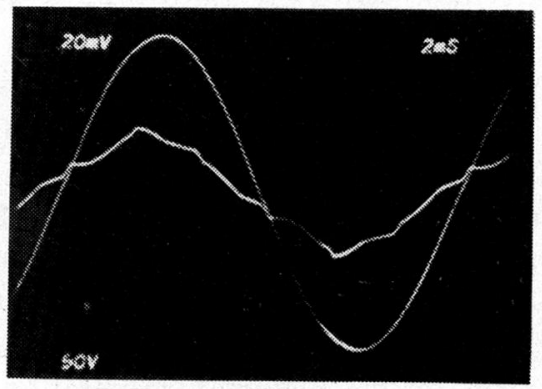

(b)

Fig. 22. Voltage and current waveshapes of CPU system 3. (a) Load side. (b) Line side.

it is near impossible to model. The randomness of the loads is due to the fact that not all of the equipment is on all the time, and equipment is constantly being added, replaced, or removed. Some help is available in knowing the characteristics of particular types of rectifiers, and from these characteristics some prediction of the distribution system response can be assumed. But the adequacy of the system should be verified through oscilloscope waveform analysis.

For any waveform analysis of a random system at least two separate locations in a distribution system must be included, and the voltage and current waveforms are absolutely essential. 
The existence of a voltage disturbance can then be correlated with current switching if a voltage spiking problem is observed. In this way actual problems and their source can be eliminated from imagined problems. The imagined problem in the three systems of this study was the "power system" which was responsible for causing the steady-state spiking. The waveforms presented clearly show that the steady-state spiking was generated within each system, and that the "power system" was only responding to the current switching.

The actual switching phenomena on the low-voltage side of the power supplies $(5 \mathrm{~V}, 10 \mathrm{~V}$, etc.) was not considered. It is just the presence of the stepdown transformers which produces the strange current waveshapes, especially the $\mu_{-i}$ current impulse of Fig. 7(d). Although the rectifiers on the low-voltage side are not conducting the negative current during the positive voltage excursion, the high side of the transformers do provide the path. The negative current in this case is due to the other phase interacting through the neutral conductor and magnetic circuit of the transformer.

The stiffness of a system has often been proposed as a criterion for the possibility of disturbances in solid-state switching systems. In these low-voltage $(120 / 208 \mathrm{~V})$ systems the disturbance is dependent upon the current switching producing an impulse $\mu$. And the governing criteria for the magnitude of the disturbance is the percent of full-load current that the switching represents to the system. In system number 1 the $150 \mathrm{~A}$ load represents five percent of the $500 \mathrm{kVA}$ transformer full-load capacity. System number 2 loading was approximately 33 and 50 percent of the $R P$-5 and $R P$-5a transformer capacity, as seen in Fig. 17. And for system number 3 it was 16 percent for CPU system 1 and 33 percent for CPU systems 2 and 3 . A qualitative comparison is difficult since the number and magnitude of the impulses $\mu_{ \pm i}$ are different, but qualitatively the $500 \mathrm{kVA}$ transformers are much less disturbed than the $45 \mathrm{kVA}$ transformer.

The isolation transformers have excessive voltage distortion in comparison with the distribution transformers. This is most pronounced in the second and third systems. It is for the reason that isolation transformers, with electrostatic shielding, prevent the transmission of high-frequency voltage noise into an isolated system, that they become more susceptible to voltage generation due to current impulses.

The mechanism of the distortion generation can be seen in Fig. 23. An ordinary unshielded transformer, Fig. 23(a), contains capacitive coupling between windings $C_{1}(122: 9)$. $C_{1}$ is the source of high-frequency voltage coupling into a typical power system. The introduction of the shield between windings effectively reduces the effect of $C_{1}$, but introduces a much more significant capacitance, $C_{2}$, (Fig. 23(b)) between the secondary winding and the shield. The equivalent circuit with $C_{2}$ is shown in Fig. 23(c), where the parallel inductancecapacitance $(L C)$ circuit again introduces the condition for voltage generation during the current impulse period [5].

\section{CONCLUSION}

Low-voltage power systems which have a significant percentage of or are entirely dedicated to small rectifier loads can

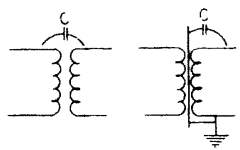

(a) (b)

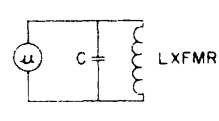

(c)
Fig. 23. Equivalent circuits for the isolation transformer. (a) Capacitive coupling between windings. (b) Shielding between windings to reduce coupling. (c) Equivalent circuit for the impulse generator.

become susceptible to generating internal voltage distortions. The primary distortion is generated by current impulses across a transformer inductance, or in the case of electrostatic shielding, across a parallel $L C$ circuit. The severity of the distortion is directly related to the percent of full-load current capacity that the impulse $\mu$ represents to the system. It is apparent that some of the practices for reducing voltage disturbances and distortions are expensive and generally counterproductive. Using transformers that are oversized in order to provide a "stiff" system and isolation transformers are two such ineffective methods.

All rectifiers have their own characteristic waveforms. Certain types can produce current waveforms which cause harmonic neutral currents that are higher than line currents on three-phase four-wire systems and require wire and transformer sizing accordingly. Preventative and corrective measures to minimize any disturbances require verification through waveform analysis of voltage and current with the use of the spectrum display to verify any questionable waveforms.

Since small loads are easily installed or moved, the problem of when certain types of rectifiers will produce nuisance spiking or hazardous current waveshapes will be random. There are several possible approaches to protect the locations where a concentration of these devices are likely to occur, such as commercial or research facilities. Some of the possible protective measures in descending order of cost could be the following.

1) Oversize all neutral conductors and transformers on designated circuits.

2) Encourage or require the facility, owners, or manager to periodically or constantly monitor the current waveforms.

3) Encourage or require end item manufacturers to provide waveform corrective devices.

4) Require rectifier manufacturers or suppliers to insure that none of the current waveforms described in this paper are produced. It is recognized that the economics of the situation will have considerable bearing on which method, if any, is used, but dissemination and awareness of the possible conditions should be emphasized.

\section{REFERENCES}

11) S. B. Dewan and A. Straughen. Power Semiconductor Circuits. New York: Wiley, 1976.

[2] J. H. Galloway, "Harmonic line currents in large thyristor six-pulse converters," IEEE Trans. Ind. Appl., vol. IA-11. pp. 256-262, May/ June. 1975.

[3] P. W. Franklin, "A theoretical study of the three phase salient pole type generator with simultaneous AC and bridge rectified DC Output-Part I 
and II," IEEE Trans. Power App. Syst., vol. PES-91, pp. 543-557, Sept./Oct., 1972.

[4] V. H. Jones and W. J. Bonwick, "Three-phase bridge rectifiers with complex source impedances," Proc. IEE, vol. 122. pp. 630-636, June 1975.

[5] G. V. Lago and D. L. Waidelich, Transients in Electrical Circuits. New York: Ronald, 1958.

[6] A. A. Mahmoud, C. Calabrese, and J. R. Tudor, Critical load protection from transient surges," IEEE Trans. Power App. Syst., vol. PES 90, pp. 1546-1554, July/Aug., 1971.

[7] L. J. Powell, Jr., "Influence of third harmonic circulating currents in selecting neutral grounding devices," IEEE Trans. Ind. Appl., vol. IA-9, pp. 672-679, Nov./Dec., 1973.

[8] P. Chowdhuri and D. D. Bock, "Coordination of transient-voltage characteristics of electrical systems, " IEEE Trans. Ind. Appl., vol. IA-9, pp. 577-581.

[9] H. W. Ott, Noise Reduction Techniques in Electronic Systems. New York: Wiley, 1976.

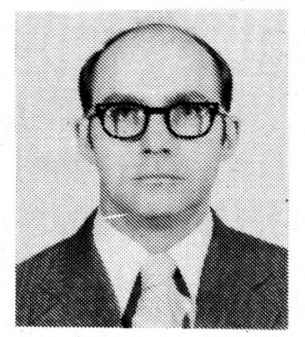

William P. Gibbons (S'75-M'76) received the A.E. degree in electrical and electronic technology from Pennsylvania State University, McKeesport, PA, in 1968; the B.S.E.E. degree from the University of Missouri, Columbia, MO, in 1971; and the M.S.E.E. degree from the Air Force Institute of Technology. Wright-Patterson AFB, OH in 1976.

His technical background includes both employment in manufacturing and military experience. After completing an initial military obligation and attending Penn State he worked for the E. L. Wiegand

Company, Pittsburgh, PA, in manufacturing quality control. His assignments since recall to active duty have been in aircraft maintenance, communication, and electrical power system design for research and development projects, and he is presently the Environmental Planner for Osan AB, Korea. While assigned to Korea he participated in off-duty educational programs as a part-time instructor for the University of Maryland, Far East Division, and the Los Angeles Community Colleges, Overseas. 